INRA Prod. Anim., 2010, 23 (1), 81-90

\title{
Incertitude et adaptation : cadres théoriques et application à l'analyse de la dynamique des systèmes d'élevage
}

B. DEDIEU, S. INGRAND

INRA, UMR1273 Mutations des Activités, des Espaces et des Formes d'Organisation dans les Territoires Ruraux, F-63122 Saint-Genès Champanelle, France AgroParisTech, Mutations des Activités, des Espaces et des Formes d'Organisation dans les Territoires Ruraux, F-75231 Paris, France CEMAGREF, Mutations des Activités, des Espaces et des Formes d'Organisation dans les Territoires Ruraux, 24 avenue des Landais F-63172 Aubière, France VetAgroSup, Mutations des Activités, des Espaces et des Formes d'Organisation dans les Territoires Ruraux, Site de Marmilhat, F-63370 Lempdes, France

Courriel :dedieu@clermont.inra.fr

L'incertitude croissante qui caractérise le contexte politique, économique et climatique dans lequel évolue l'élevage rend incontournable l'analyse et l'évaluation des capacités d'adaptation des systèmes d'élevage, c'est-à-dire de leurs capacités à résister à des perturbations de toute nature et à s'inscrire dans une dynamique d'évolution sur le long terme. Les concepts de flexibilité et de résilience permettent de guider l'exploration des fondements de ces capacités d'adaptation tant en fermes que via la modélisation.

Les recherches sur l'élevage sont depuis toujours guidées par la recherche d'une amélioration de l'efficacité de l'activité, notamment en explorant des solutions optimisées sur des critères de marge brute ou de revenu et en analysant des marges de progrès technique des systèmes en place vers ces optimums. L'incertitude croissante qui caractérise le contexte politique, économique et climatique dans lequel évolue l'élevage actuellement, suggère à des auteurs de plus en plus nombreux, d'analyser et d'évaluer les systèmes d'élevage en termes de capacité d'adaptation, que nous définissons par «la capacité à résister à un ensemble hétérogène de perturbations sur le moyen terme et à s'inscrire dans une dynamique, un mouvement, qui permette de durer sur le long terme» (Mignon 2001, Dedieu 2009, Ingrand et al 2009), alors que :

- les données concrètes décrivant l'avenir lointain sont inconnues ;

- l'occurrence de crises graves et soudaines (sanitaires et financières) est plus que probable sans être probabilisable ;

- l'instabilité des prix (produits, intrants) et l'orientation générale des réformes de la PAC (moins de filets de sécurité) rendent l'environnement éco- nomique de l'élevage plus incertain et les garde-fous face aux aléas de prix moins nombreux et moins efficaces que par le passé ;

- il y a un risque accru d'occurrence d'événements climatiques extrêmes en lien avec le réchauffement climatique.

Les solutions proposées jusqu'alors pour faire face aux aléas, dans le cadre du modèle productiviste dominant, font l'objet de critiques : irrigation, pesticides, prévention sanitaire poussée etc., principalement en raison de leurs effets négatifs sur l'environnement. Mais la recherche de modèles de production à haute valeur environnementale implique également, en élevage herbivore, la conception de systèmes qui puissent s'accommoder des aléas, notamment climatiques.

La zootechnie des systèmes d'élevage est une discipline qui permet de rendre compte de la façon dont sont articulées les dimensions humaines et techniques de l'activité d'élevage (Dedieu et al 2008a). Nous proposons dans cet article d'illustrer ce que recouvrent les recherches sur les capacités d'adaptation, en référence à ce cadre conceptuel de système d'élevage. Dans un premier temps, nous préci- sons à quelle vision dynamique de l'élevage nous nous rattachons, en référence à la littérature traitant d'une part de résilience et d'autre part de flexibilité. Ces concepts sont développés dans d'autres disciplines que la zootechnie, mais ont comme point commun de permettre de rendre compte du comportement de systèmes dans un environnement soumis en permanence à des perturbations. Puis, nous illustrons dans une deuxième partie, comment nous appliquons ces concepts à l'analyse des logiques d'action des éleveurs sur le long terme et à l'étude des modalités de résistance aux aléas à plus court terme. Nous résumons et discutons dans une troisième partie la façon dont la modélisation explore les capacités d'adaptation des systèmes d'élevage (Dedieu et al 2008b).

\section{1 / Cadres d'analyse de la dynamique des systèmes d'élevage}

\section{1 / Les dynamiques sur le long terme \\ Quelle vision dynamique de l'élevage et quelle représentation de l'adaptation}


sous-tendent les recherches en zootechnie ? Avec la prégnance des démarches d'optimisation technico-économique dans les raisonnements de la zootechnie (Landais et Bonnemaire 1996), il s'agit pour l'essentiel de produire des connaissances et des modèles pour améliorer la performance d'une situation donnée ou pour rechercher de nouvelles combinaisons d'activités et/ou de techniques qui soient cohérentes avec un nouveau contexte (une réforme de la PAC par exemple (Veysset et al 2005), mais aussi une nouvelle réglementation environnementale, de nouveaux rapports de prix, de nouveaux marchés...). Dans ces approches, la vision dynamique des systèmes d'élevage correspond à une succession de passages d'un état donné du système à un instant $t$ (caractérisé par un agencement entre des décisions d'éleveur et un fonctionnement biotechnique du troupeau) vers un nouvel état à un instant $t+1$. Le changement d'état est le plus souvent justifié par un changement survenu dans l'environnement. Aux instants $t$ et $t+1$, les états du système sont considérés comme stables, du moins à l'état d'équilibre, tout comme leur environnement.

D'autres façons de considérer la dynamique des systèmes de production agricole sur le temps long ont vu le jour depuis une vingtaine d'années. Lev et Campbell (1987), par exemple, soulignaient le danger d'ignorer les interactions de long terme dans la recherche de solutions plus viables pour les agriculteurs et pointaient l'enjeu de maintenir de la flexibilité dans des systèmes soumis à l'incertitude. Pour Grosseti (2006), «on peut très bien faire de la science (de la nature) en acceptant des parts d'imprévisibilité, en faisant de la contingence [des événements perturbants, d'ordre interne ou externe] une place qui n'est pas celle des résidus [des statisticiens]». Les écologues comme Holling (1973) considèrent que la mise en dynamique d'un système inclut des chocs brutaux, qui peuvent remettent en cause son existence et qui font partie de tout un ensemble de perturbations quasi permanentes avec lesquels le système doit composer. Deux éléments se dégagent de ces travaux :

- l'adaptation est vue comme une capacité à faire face à un ensemble de perturbations permanentes et de nature variée, selon qu'elles sont prévisibles ou non, sévères ou modérées ;

- les acteurs, pour gérer les changements de court terme, intègrent des éléments d'une vision à plus long terme, laquelle inclut l'idée de préserver des capacités d'adaptation dans un contexte incertain.

Figure 1. Le cycle adaptatif d'un système socio-écologique (Holling 2001).

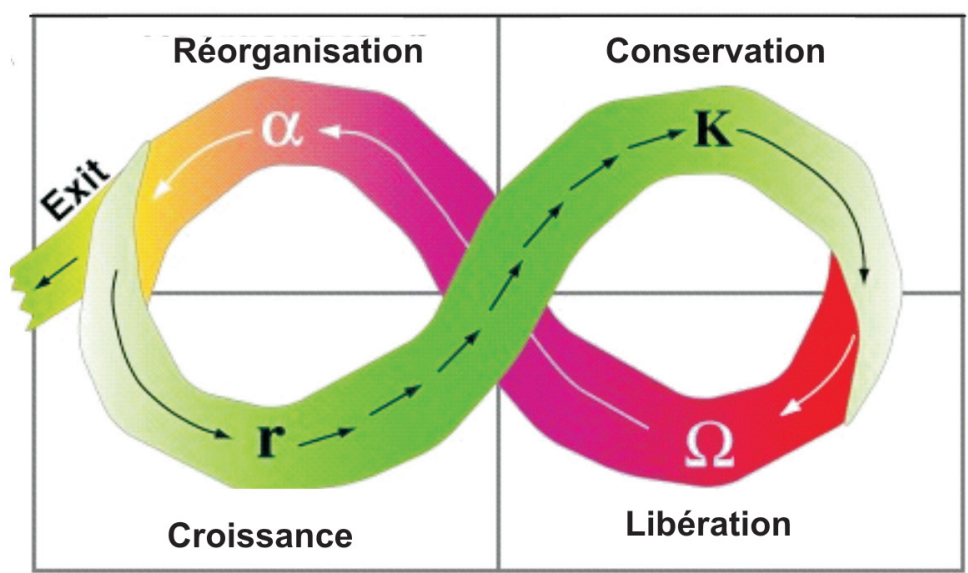

$\mathrm{r}$ à $\mathrm{K}$ : une situation qui change peu ou lentement, avec des perturbations modérées. L'apprentissage permet une capacité de contrôle et de maîtrise croissante.

$\mathrm{K}$ à $\Omega$ : choc. Libération des ressources.

$\Omega$ à $\alpha$ : mobilisation du potentiel (ressources, capital) pour réorganiser le système.

Après $\alpha$ : Exit (échec) ou reprise d'un cycle nouveau.

Holling (2001) a proposé un cadre général de représentation de la dynamique d'un système socio-écologique qui regroupe ces deux idées. La dynamique est représentée par un enchaînement de cycles qualifiés d'adaptatifs (figure 1). Chaque cycle est composé de phases successives au cours desquelles le système subit soit des perturbations modérées associées à une maîtrise croissante du système avec l'expérience, soit des chocs nécessitant de reconfigurer le système. Si cette reconfiguration échoue, le système s'effondre.

\section{2 / Quelques concepts pour traiter de l'adaptation des systè- mes d'élevage à des perturba- tions en continu}

Sauvant et Martin (2010), dans ce numéro, proposent un certain nombre de concepts et de critères rendant compte de la résistance aux perturbations d'entités relevant du domaine de la zootechnie (animal, système de production). Pour notre part, et concernant les approches au niveau des systèmes d'élevage en ferme, nous proposons essentiellement deux concepts : résilience et flexibilité. Chacun d'entre eux donne lieu à une littérature spécifique et abondante dont nous ne ferons pas état ici, d'autant qu'ils sont pour l'essentiel mobilisés dans d'autres disciplines et sur d'autres objets que les systèmes d'élevage (Astigarraga et al 2008, Ingrand et al 2009).

\section{a) La résilience}

La résilience est un concept utilisé dans de nombreuses disciplines notamment en écologie mais aussi en psychologie (Cyrulnick 2001) ou en physique. Il permet de rendre compte :
- de la résistance d'un système aux chocs (Walker et al 2004). L'enjeu principal, dans le cas de chocs brutaux mettant en péril le système, est de mobiliser les bonnes ressources permettant sa reconfiguration ;

- dans une vision plus étendue, de la capacité d'un système à perdurer, c'està-dire à être capable de faire face à des perturbations à toutes les étapes du cycle adaptatif.

Tous les auteurs ne mettent pas l'accent de façon équivalente sur ces deux points, la résistance au choc étant cependant plus détaillée car fondatrice du concept. La mobilisation du concept à l'échelle de l'exploitation agricole relève plutôt de la deuxième définition (Milestad et Darnhofer 2003 ; Darnhofer et al 2010).

Selon Gunderson (2000), trois stratégies permettent d'accroître le niveau de résilience des systèmes pilotés :

- l'accroissement du pouvoir tampon du système (marges de manœuvre) ;

- l'accroissement du caractère adaptatif du pilotage en jouant sur différents niveaux d'échelle (spatiale et temporelle) ;

- la création des conditions d'émergence d'innovations (sources de changements des caractéristiques des systèmes, capacité d'apprentissage).

\section{b) La flexibilité}

La flexibilité est un concept des sciences de gestion et de l'économie industrielle (Chia et Marchenay 2008). Elle renvoie à l'image «du roseau qui plie mais ne rompt pas» (La Fontaine). Nous rappelons ici deux définitions de la flexibilité d'une organisation : 
- «son aptitude à s'accommoder aux circonstances, à absorber des changements, sa capacité à préserver et à créer des options, à apprendre» (Reix 1979, Pasin et Tchokogué 2001) ;

«des procédures qui permettent d'accroître la capacité de contrôle sur l'environnement, de diminuer la sensibilité du système à son environnement» (Astigarraga et al 2008). Cette définition amène à considérer le degré de proactivité du système information, décision pour anticiper et réagir à l'occurrence d'aléas. Ces auteurs précisent en outre que la flexibilité est une propriété qui n'est pas mesurable en soi, mais qui dépend du contexte (le type d'aléas pris en compte) et des buts recherchés.

La flexibilité peut s'envisager à des échelles temporelles variées. Ainsi la flexibilité stratégique est la «capacité à modifier la structure, les ressources et les compétences de l'entreprise pour $s$ 'adapter ou devancer les évolutions de l'environnement». La flexibilité opérationnelle concerne plutôt l'«ajustement au cours du cycle de production à des aléas divers» (Tarondeau 1999). Ce dernier distingue les sources de flexibilité internes, relatives au système de production et externes, en lien avec les réseaux de relation sociaux et économiques. Les sources internes sont déclinées en flexibilité «inputs, processus, produits»». L'encadré 1 propose une reformulation de ces sources internes dans le domaine de l'élevage bovin allaitant.

A l'instar de Gunderson (2000) pour la résilience, Aaker et Mascarenhas (1984) proposent plusieurs leviers pour développer la flexibilité d'une organisation :

- la diversification des processus, des activités et des produits, incluant l'élargissement de la gamme, mais aussi la participation à différents marchés ;

- l'accroissement de l'autonomie de chaque unité de production par rapport aux autres ;

- le développement de ressources potentiellement utiles, c'est-à-dire non mobilisées en permanence, mais mobilisables «au cas où»: redondances fonctionnelles, compétences non exploitées, marges de manœuvre.

c) Flexibilité et résilience : des concepts finalement assez proches

Tels que sommairement présentés, les concepts de «résilience» et «flexibilité»» présentent de nombreuses similitudes :

- ils rendent compte du comportement de systèmes soumis en continu à des perturbations, certaines pouvant être
Encadré 1. Les sources internes de la flexibilité : illustrations en élevage d'herbivores (domaines technique et travail)

- Inputs : des spécifications produits et processus révisables (par exemple règles d'ajustement de la voie femelle en bovin viande, du choix des dates de sevrage, de la durée des sessions de reproduction ; règles d'ajustement de la répartition du travail et du contenu des tâches

- Des processus avec des ressources potentiellement utiles, pas trop spécialisées et des capacités tampon. Par exemple : cultures de céréales autoconsommés ou vendues selon l'état des stocks : un chargement faible pour limiter l'impact d'aléas climatiques ; la préparation de pâtures de "soudure» pour passer des périodes difficiles (mise à l'herbe, attente des pluies d'automne) ; des stocks sécuritaires ; plusieurs périodes de mises bas dans l'année pour diversifier le positionnement des cycles productifs dans le calendrier ; plusieurs périodes de conservation des femelles de renouvellement dans l'année ; une polyvalence des travailleurs et des formes d'organisation du travail stables, générant des marges de manœuvre

- Des produits diversifiés : types de produits animaux, périodes de vente.

des chocs nécessitant des reconfigurations. La vision dynamique de long terme est celle d'une co-évolution du système et de son environnement (Darnhofer et al 2010) ;

- ils différencient différents horizons temporels : le long terme des cycles adaptatifs, de la succession de phases de maîtrise croissante puis de reconfiguration du système versus le court terme de la flexibilité opérationnelle ;

- ils proposent des pistes pour accrô̂tre la capacité d'adaptation des systèmes, avec les notions de pouvoir tampon, de marges de manœuvre, de gestion adaptative, d'apprentissage, etc.

\section{2 / Application à l'analyse en ferme des systèmes d'éle- vage d'herbivores}

Les travaux développés depuis quelques années sur l'adaptation des systèmes d'élevage en ferme se scindent en deux catégories, relevant de deux questions. La première porte sur les moyens de formaliser les «logiques d'action sur le long terme» sur la base de l'étude des modalités d'adaptation de systèmes présentant des propriétés apparentes de résilience. La seconde porte sur les moyens de formaliser les sources de flexibilité opérationnelle des systèmes d'élevage, pour des aléas spécifiés, et à tester l'hypothèse selon laquelle ces sources sont liées à des modalités spécifiques d'adaptation des systèmes sur le long terme (Dedieu et al 2008b).

\section{1 / Les logiques d'action sur le long terme}

L'application en élevage des cadres théoriques présentés précédemment permet d'analyser «l'action en situation d'incertitude» (Lemery et al 2005), autrement dit de produire des connaissances sur la dynamique des systèmes au travers de l'étude des logiques d'action sur le long terme (Bernoux 1996). Ces logiques sont construites sur la base d'un certain nombre de principes qui guident l'action sur le plan technique, économique, financier et humain, et qui, dans les circonstances propres à chaque exploitation et selon la nature et l'intensité des perturbations internes ou externes, vont se matérialiser par la trajectoire d'évolution du système familleexploitation (Osty 1978).

\section{a) Démarche et méthode}

Nous avons réalisé entre 2002 et 2008 plusieurs opérations de recherches visant à 1) formaliser les principes d'action (nature, modalités) dont les éleveurs font état pour expliquer l'évolution de leur système ; 2) identifier la diversité des logiques d'action (ou chemins pour durer), qui permettent aux éleveurs de tenir sur le long terme. Ces opérations de recherche ont concerné (tableau 1) l'élevage bovin viande en Bourgogne, l'élevage laitier dans le Ségala (Massif Central), et pour sortir d'un contexte de fort encadrement de la production, l'élevage bovin (viande et lait) en Uruguay. Ce pays d'économie ultralibérale a connu ces trente dernières années, deux dévaluations, une crise sanitaire grave (fièvre aphteuse, le pays étant exportateur à $80 \%$ ) et des accidents climatiques (sécheresses, inondations). Il permet d'envisager des situations où les incertitudes sont plus importantes que dans un pays européen et les filets de sécurité moindre.

Ces opérations ont mobilisé un groupe de chercheurs associant zootechniciens, gestionnaires et, sauf en Uruguay, des sociologues, en partenariat avec des professionnels locaux (Chambre d'agriculture de Saône et Loire, Contrôles laitiers du Cantal et du Lot, Institut Plan Agropecuario d'Uruguay). La méthodologie consistait à réaliser des entretiens non directifs visant au recueil des éléments permettant de reconstituer les trajectoires du système famille-exploitation-autres activités sur un petit échantillon (39 exploitations au total), constitué sur la base de critères considérés comme favorables ou défavorables à la 
Tableau 1. Trois opérations de recherches sur «l'action en situation d'incertitude» en élevage herbivores.

\begin{tabular}{|l|c|c|c|l|}
\hline & $\begin{array}{c}\text { Nombre } \\
\text { d'exploitations }\end{array}$ & Période & $\begin{array}{c}\text { Dimensions } \\
\text { des troupeaux }\end{array}$ & Publication \\
\hline $\begin{array}{l}\text { Bovin viande } \\
\text { Bourgogne }\end{array}$ & 14 & $2001-2004$ & $\begin{array}{c}40-145 \\
\text { vaches }\end{array}$ & $\begin{array}{l}\text { Lemery et al } \\
2005\end{array}$ \\
\hline $\begin{array}{l}\text { Bovin lait } \\
\text { Ségala }\end{array}$ & 14 & $2006-2009$ & $\begin{array}{c}17-55 \\
\text { vaches }\end{array}$ & $\begin{array}{l}\text { Begon et al } \\
2009\end{array}$ \\
\hline $\begin{array}{l}\text { Bovin } \\
\text { Uruguay }\end{array}$ & 11 & $2006-2007$ & $\begin{array}{c}8 \text { allaitants : } \\
77 \text { à } 4300 \text { têtes } \\
\text { 3 lait : } \\
140-3500 \text { têtes }\end{array}$ & $\begin{array}{l}\text { Levrouw et al } \\
2007\end{array}$ \\
\hline
\end{tabular}

résilience par nos partenaires. L'analyse des données individuelles a été effectuée selon le cadre proposé par Moulin et al (2008). Il s'agissait de délimiter :

- des phases de «cohérence», associés à l'expression d'un ensemble de principes d'action et d'une dynamique du système marquée par des perturbations ne nécessitant pas de reconfiguration du système ; elles correspondent aux phases de maîtrise croissante du cycle adaptatif de Holling (2001) ;

- des phases de rupture associées à des chocs (de toute nature, interne ou externe) débouchant sur une reconfiguration du système. L'interrogation porte alors sur le renouvellement ou non des principes d'action de la phase précédente.

L'analyse a été réalisée terrain par terrain et sur l'ensemble des 39 cas. Nous avons construit à l'aide de l'outil graphique de Bertin (1977), une typologie des logiques d'action, en croisant les exploitants (individus) et leurs principes d'action (variables et modalités). Les résultats des analyses ont été systématiquement présentés et discutés avec les éleveurs enquêtés lors de réunions collectives (tous les terrains) et d'un retour individuel (Ségala).

\section{b) Les différentes logiques identifiées}

La mise en commun des données provenant des trois études met en exergue 5 principes guidant les évolutions dans la trajectoire des exploitations, que les éleveurs mettent en avant pour les expliquer et/ou les justifier :

la configuration du système famille-exploitation, avec deux principes relatifs $i$ ) à la taille : avec des modalités "être gros pour tenir» ou au contraire «s'agrandir, c'est pas pour moi : on $s^{\prime} y$ perd»; ii) à la combinaison d'activités, opposant la diversification «pas tous les oufs dans le même panier» et la spécialisation «pour être compétent et efficace»;

- la prise de risques financier ou technique, pour l'amélioration du système, avec les trois modalités : «jamais», «nécessaire», «que s'il est très contrôlé»;
- les finances, c'est-à-dire le rapport à l'endettement (jamais, ou mal nécessaire), à l'épargne (systématique ou non), à la possibilité d'ajuster les prélèvements familiaux en année difficile ;

le fonctionnement du système technique, avec trois modalités correspondant à l'accent mis par les éleveurs sur des dimensions différentes de ce fonctionnement : une ambition technique forte («c'est le garant pour tenir») ; une ambition gestionnaire («ce qui importe, c'est l'optimisation de l'ensemble des ressources de l'exploitation, y compris d'un travail maîtrisé»» ainsi que du volet fiscal) ; la volonté de al 2009) temps haut niveau de performances recherché ; significatif ; tionnelle extensive coût/bénéfice. conserver des souplesses dans le processus de production («en garder sous la pédale», «ne jamais être à fond») ;

- les réseaux sociotechniques (d'information, d'échanges et de conseil, permettant la maîtrise de l'aval («il est nécessaire d'investir dans des organisations de producteurs》).

Tous ces principes ne jouent pas le même rôle dans le choix des différents chemins sur le long terme, échantillon par échantillon. Les principes liés à la configuration du système familleexploitation et au fonctionnement du système technique semblent avoir un poids relatif plus important que les autres. Les encadrés 2, 3 et 4 présentent la diversité des logiques d'action sur le long terme respectivement dans le Ségala (lait), l’Uruguay (lait et viande) et la Bourgogne (viande).

Ces logiques présentent de grandes similitudes selon les terrains, sans que la situation particulière de l'Uruguay ressorte. Si les logiques ne sont pas redondantes dans les 3 terrains, il n'y a pas de logique qui ne soit représentée que dans un seul. Intensifier fortement, diversifier les activités, développer

Encadré 2. Les logiques d'action sur le long terme en élevage laitier du Ségala (Begon et

- Etre techniquement performant (indicateur : niveau de production par VL) : Peu de modification surfaces, spécialisation rapide en début de trajectoire, conjoint travaille à l'extérieur ou pas. Certains éleveurs évoluent vers des raisonnements gestionnaires avec le

- Etre «gros» en lait : Agrandissement (par agglomération) des surfaces ; augmentation des quotas, du cheptel ; tendance à la spécialisation ou tendu vers l'idée de la spécialisation laitière, (même si lors de certaines phases, il peut y avoir plusieurs activités agricoles

- Avoir un gros troupeau laitier mais également une autre activité herbivore qui fait «tampon» en cas de coup dur : Agrandissement ou gros au départ, plusieurs activités en parallèle mais lait dominant ; ambition technique lait puis «préoccupation gestionnaire» ;

- Diversifié «entrepreneur» : être gros avec plusieurs activités d'égale importance. "Faire des affaires», pas forcément faire au mieux, avec plusieurs fers au feu et chacun

- Diversifié «sur opportunités locales» : Petites structures ; essais de diversification ou de cueillette ; de la souplesse dans la conduite du troupeau laitier, sans grande ambition quant au niveau de production laitière, variable et dépendante des ressources fourragères.

Encadré 3. Logiques d'action en élevage bovin d'Uruguay (Levrouw et al 2007)

- Diversifié-bricole sur base locale : diversification des activités agricoles, pas d'agrandissement (surfaces et cheptel) ; pas d'investissements ; ajustements continus des activités et des itinéraires techniques, avec une conduite traditionnelle (extensive) du cheptel

- S'agrandir : spécialisation en élevage bovin allaitant ; agrandissement (cheptel et surfaces) ; investissements sous condition d'une épargne préalable ; conduite d'élevage tradi-

- Optimiser le système technique : spécialisation en élevage bovin viande ou lait, avec recherche de haut niveau de productivité animale ; endettement pour investir dans la technologie ; recherche permanente d'innovation y compris avec prise de risque

- Contrôle maximum : spécialisation en lait ou viande avec recherche de haut niveau de productivité ; agrandissement (surfaces et cheptels) ; investissements financiers lorsque l'épargne est suffisante et la prévisibilité du retour claire. Innovation raisonnée avec calcul 
Encadré 4. Flexibilité opérationnelle de systèmes d'élevage Bourguignon, selon les types de logiques d'action sur le long terme (Lemery et al 2005). Les aléas considérés : baisse du prix de bovins, sécheresse. Tensions fortes -- ; flexibilité forte ++

\section{- Maîtrise technique par optimisation + investissement dans le collectif (aval)} Caractéristiques des exploitations : production de taurillons 18 mois ; chargement élevé (supérieur à 1,8 UGB/ha SFP) ; vêlages précoces ; vente en groupement ; petites surfaces.

Tensions et souplesses (flexibilité + ou -) : un seul produit et un chargement élevé flex tech-éco interne -- ; vente en groupement, qui aide en période difficile (par exemple achats groupés de fourrages en cas de sécheresse) flex tech-éco externe ++ ; petite surface en couple flex travail + .

\section{- S'agrandir}

Caractéristiques des exploitations : grandes surfaces, production de broutards, un peu de finition selon l'année, chargement moyen.

Tensions et souplesses (flexibilité + ou -) : un produit dominant mais jeu sur la voie femelle (engraissement ou non), chargement flex tech-éco interne + . Charges de travail élevées flex travail -.

\section{- Garder de la souplesse, être autonome}

Caractéristiques des exploitations chargement faible $(<1,2$ UGB/ha), beaucoup d'acheteurs d'animaux (maquignons), plusieurs produits animaux (bœufs maigres, gras, broutards plus ou moins repoussés), avec une répartition variable selon les années. Taille moyenne à grande. Principe d'autonomie sur tous les plans.

Tensions et souplesses (flexibilité + ou - ) : chargement faible, plusieurs produits : flex tech-éco interne ++ , plusieurs acheteurs flex tech-éco externe ++ ; autonomie très forte dans le travail flex travail.

\section{- Diversifier}

Caractéristiques des exploitations : le dimensionnement de l'activité bovine peut varier produits bovins changeants d'une année à l'autre, chargement et surfaces intermédiaires. Tensions et souplesses (flexibilité + ou -) : ajustement des produits selon les cours et les stocks, chargement flex tech-écon interne +, concurrence entre activités pour le travail flex travail.

l'activité d'élevage ou une combinaison d'activités valorisant les ressources locales, s'agrandir (dans un cadre extensif ou intensif) sont bien des logiques d'action qui semblent candidates à la généralisation. L'incrémentation de la base de données se poursuit sur d'autres terrains (Argentine, Alpes du Nord), dans le cadre d'études en cours et tend à confirmer ces premières observations (Cialdella et Dedieu 2010).

\section{2 / Les logiques d'action et la flexibilité opérationnelle}

Nous avons appliqué la grille d'analyse de la flexibilité opérationnelle de Tarondeau (1999) aux terrains français en référence aux aléas climatiques et à la volatilité des prix.

Un premier résultat est qu'il apparaît nécessaire de considérer la flexibilité externe (ou «relationnelle», Chia 2008), pour comprendre la flexibilité interne des systèmes d'élevage. Par exemple, en Bourgogne, le système très intensif avec production de taurillons de 18 mois, a priori très sensible aux aléas de prix (beaucoup d'intrants, spécialisation des catégories d'animaux vendus) et de climat (niveau de chargement élevé : 1,8 UGB (Unités Gros Bovins)/ha dans notre échantillon), sans grande marge de manœuvre sur les itinéraires techniques (les croissances doivent être soutenues, les ventes sont programmées), possède finalement une réelle capacité à résister qui vient du fait qu'il est soutenu par une organisation collective (groupement de producteurs) en cas de coup dur.

Un deuxième résultat est que la composante travail est importante à considérer pour rendre compte de la flexibilité interne des systèmes. Par exemple, le système très autonome extensif avec production de bœufs, bénéficie de réelles surcapacités (faible chargement, stocks sécuritaires) et de la possibilité d'adaptation de la gestion, notamment du profil de vente selon les conditions de l'année (bœuf gras ou maigre, génisses vendues plus ou moins âgées). Il est très résistant aux aléas climatiques et de prix. Mais le principe d'autonomie ne s'applique pas qu'au système fourrager : il est prégnant pour l'organisation de la main-d'œuvre, essentiellement familiale. Il induit une fragilité vis-à-vis du travail, dès que l'un des travailleurs permanents fait défaut. Le même commentaire s'applique pour la logique d'agrandissement laitier avec ambition technique dans le Ségala, où les tensions de travail sont très fortes.

Le troisième résultat concerne les interactions entre les logiques d'action des éleveurs et la flexibilité des systèmes. Chaque logique d'action génère des tensions qui influent sur la façon dont le système peut résister aux aléas, et sur la façon dont l'éleveur reçoit les injonctions au changement (filières,
PAC, enjeux environnementaux). Nous illustrons dans l'encadré 4 les interactions entre la typologie des logiques d'action sur le long terme en élevage bovin viande de Bourgogne et les sources de flexibilités interne et externe des systèmes d'élevage (positive ou négati$\mathrm{ve}=$ tensions). Cet encadré montre qu'à chaque type de système, sont associés des sources et des niveaux de flexibilité très variables, sans qu'aucune solution ne soit totalement positive sur tous les plans.

Nos observations sur les terrains français montrent que les éleveurs sécurisent leurs systèmes vis-à-vis des aléas climatiques avec des surcapacités en stock fourrager (renforcés après la sécheresse de 2003), alors que cette formule de sécurisation est rare en élevage bovin allaitant d'Uruguay où le recours à la capacité des animaux à mobiliser (jusqu'aux limites vitales parfois) et reconstituer leurs réserves corporelles est plus fréquent. Elles suggèrent également que la gestion adaptative tient une place importante dans la dynamique d'évolution des systèmes. Les choix d'adaptation de la conduite peuvent porter :

i) sur le projet de production qui peut être ajusté selon les conditions de l'année : le profil de vente, la quantité de lait à atteindre, voire l'effectif d'un atelier herbivore secondaire ;

ii) sur l'adaptation des itinéraires techniques (calendrier d'alimentation, dates de sevrage, durée de la période de reproduction...).

On retrouve ici les différences de modalités concernant les principes de fonctionnement technique évoqués dans la partie $2.1 b$ : les éleveurs dont les actions sont guidées par le principe d'une ambition technique peuvent adapter les itinéraires techniques selon les conditions de l'année ; ceux qui recherchent de la souplesse sont susceptibles d'adapter, en plus des itinéraires techniques, leur projet de production. Les éleveurs aux ambitions gestionnaires n'ont pas de comportement uniforme dans notre échantillon.

\section{3 / Contribution de la modélisation à l'analyse des comportements adapta- tifs}

La modélisation systémique intègre par définition une vision dynamique $d u$ comportement du système modélisé. Elle constitue un moyen d'étude des capacités des systèmes à faire face à des perturbations, que ces dernières soient d'ordre interne (aléa biologique) ou 
externe (climat), et contribue à mettre à jour les éléments qui fondent ces capacités. Dans le domaine des systèmes d'élevage, les démarches de modélisation sont développées depuis une vingtaine d'années en France (Dedieu et al 2008a). Nous en résumons ici brièvement les principaux acquis, en fonction des finalités des travaux concernés, puis nous en discutons les limites et insuffisances au regard des observations en ferme.

\section{1 / Principaux acquis des tra- vaux de modélisation}

a) La diversité des trajectoires productives du troupeau, fondement des capacités «tampon»

Les premiers modèles développés dans les années 90 ont été spécifiquement formalisés pour rendre compte du fonctionnement et des performances de systèmes d'élevage en milieu difficile, marqués par les aléas climatiques. Il s'agissait d'expliquer la relative stabilité de ces performances techniques d'une année à l'autre en explorant les propriétés régulatrices des troupeaux découlant de conduites aux règles apparemment très stables dans le temps et dans un contexte où ces systèmes n'étaient pas sécurisés par des surcapacités en stock. Les travaux de Santucci (1991) (élevage caprin corse), Moulin (1993) (élevage de petits ruminants au Sénégal), Lasseur et Landais (1992) ainsi que Girard et Lasseur (1997) (élevage ovin méditerranéen) ont souligné que ces propriétés régulatrices sont fondées sur l'organisation de plusieurs sessions de mises bas dans l'année, permettant à des femelles infertiles à une session de participer rapidement à une autre session. La situation poussée à l'extrême, correspondant aux climats les plus aléatoires est l'organisation d'une reproduction permanente toute l'année (cas du Sénégal). Lasseur et Landais (1992) ont suggéré que cette organisation de la reproduction (et de la réforme, autorisant des épisodes d'infertilité temporaire) permettait, avec la variabilité de comportement biologique, la construction de trajectoires animales au contenu productif varié (figure 2 ). Et que cette variabilité était à l'origine de la stabilité globale des performances de troupeaux. Tichit et al (2004) ont confirmé le rôle de la diversité des trajectoires productives comme ressort de la capacité «tampon» des systèmes d'élevage. Les simulateurs de fonctionnement de troupeaux développés ultérieurement dans des contextes très intensifs (élevage ovin, caprin et porcin) où l'aléa porte sur l'occurrence d'épisodes d'infertilité (Benoit 1998, Cournut et Dedieu 2004, Martel et al 2008, Puillet 2010) confirment ces résultats obtenus en milieux pastoraux.

Figure 2. Typologie des trajectoires productives dans un élevage ovin pastoral des Préalpes ayant un niveau très régulier de performances annuelles, avec trois sessions de reproduction dans l'année pour le troupeau (Lasseur et Landais 1992).

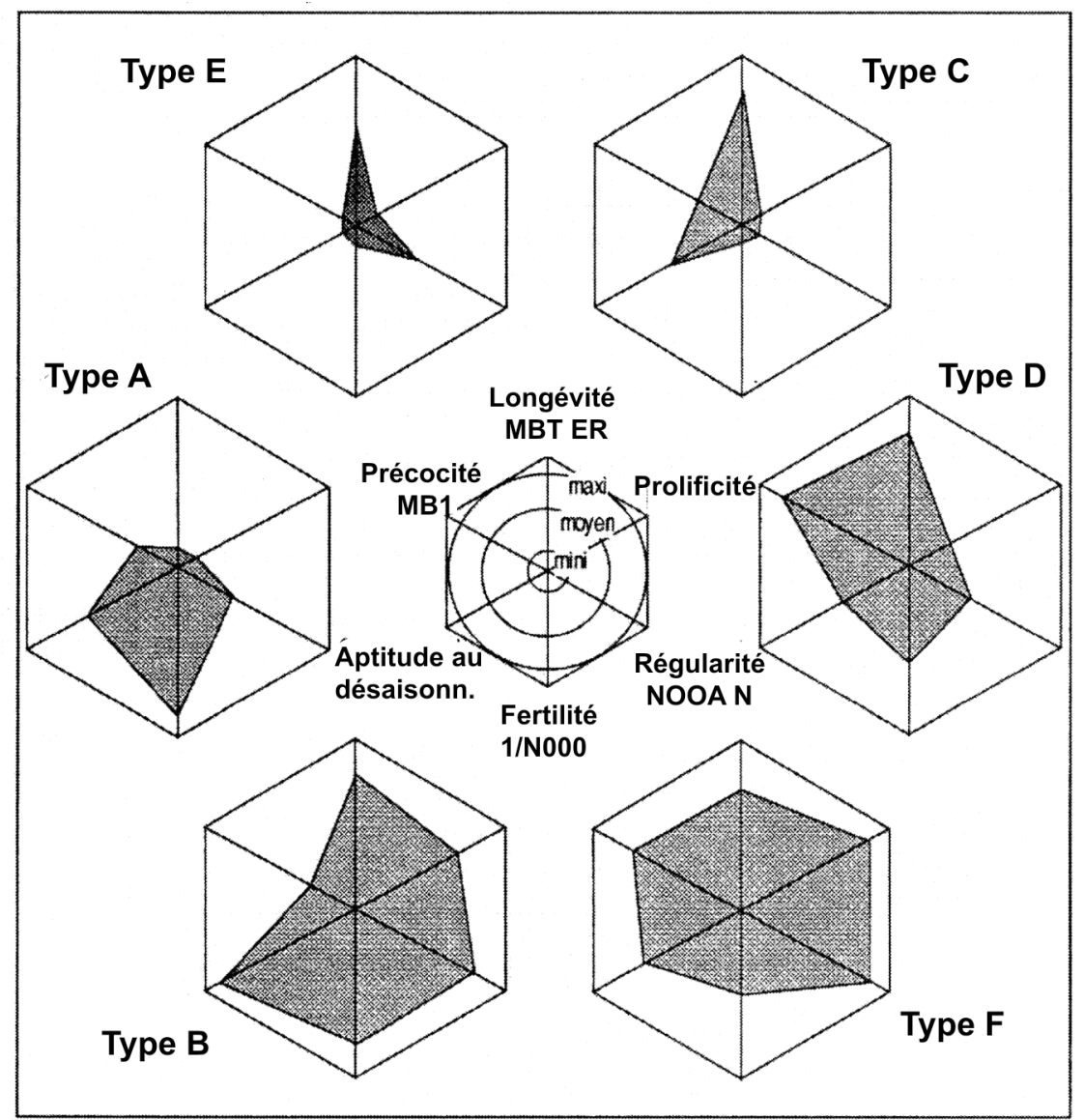

Aptitude au désaisonnement : nombre de mises bas à l'automne/nombre de mises bas. Fertilité : nombre d'épisodes d'infertilité.

Longévité : âge moyen à la dernière mise bas.

Régularité : nombre de phases avec 2 épisodes successifs d'infertilité.

Prolificité : nombre d'agneaux nés/nombre de mises bas.

Précocité : âge à la première mise bas.

\section{b) Gestion adaptative des itinéraires techniques}

Les travaux de modélisation du fonctionnement des systèmes d'élevage intégrant des modules de gestion de la production fourragère, finalisés par des enjeux combinés de production animale et d'environnement, prennent en compte la variabilité climatique et la nécessité d'adapter les itinéraires techniques d'utilisation des surfaces en fonction des sommes de températures et de la pluviométrie (Jouven et Baumont 2008, Chardon et al 2009, Vayssière et al 2009). Les simulateurs développés retiennent le cadre du modèle d'action de l'agriculteur (Sébillote et Soler 1990), comme base conceptuelle de formalisation de la gestion adaptative des itinéraires techniques. Ce cadre est construit autour d'un objectif de production, d'une planification et de rendez-vous d'ajustements au fur et à mesure du déroulement du plan, dont la première application a été le simulateur de Coleno et al (2002). Ainsi, l'aléa climatique est pris en compte dans la simulation de conduites d'élevage, sans cependant faire l'objet d'une exploration spécifique sur l'intérêt de différentes options d'adaptation.

c) Concevoir des systèmes d'élevage flexibles

Plusieurs travaux de modélisation ont explicitement pour objectif d'aider à construire un raisonnement sur la flexibilité et/ou la résilience des systèmes d'élevage. A l'aide des formalismes mathématiques de la théorie de la viabilité, Doyen et Tichit (1999) ont cherché à définir et comparer le fonctionnement de systèmes mixtes ovins-lamas de l'altiplano bolivien, soumis à un environnement très fluctuant, incluant des chocs (sécheresses sévères, vente d'une partie du troupeau pour des raisons familiales). Il s'agit en quelque sorte, en référence au cycle adaptatif de Holling (2001), de chercher à identifier les 
Figure 3. Arbre de discrimination à dires d'experts des possibilités d'utilisation d'une prairie en fonction des contraintes topographiques et d'exploitation qu'elle présente (Martin et al 2009).

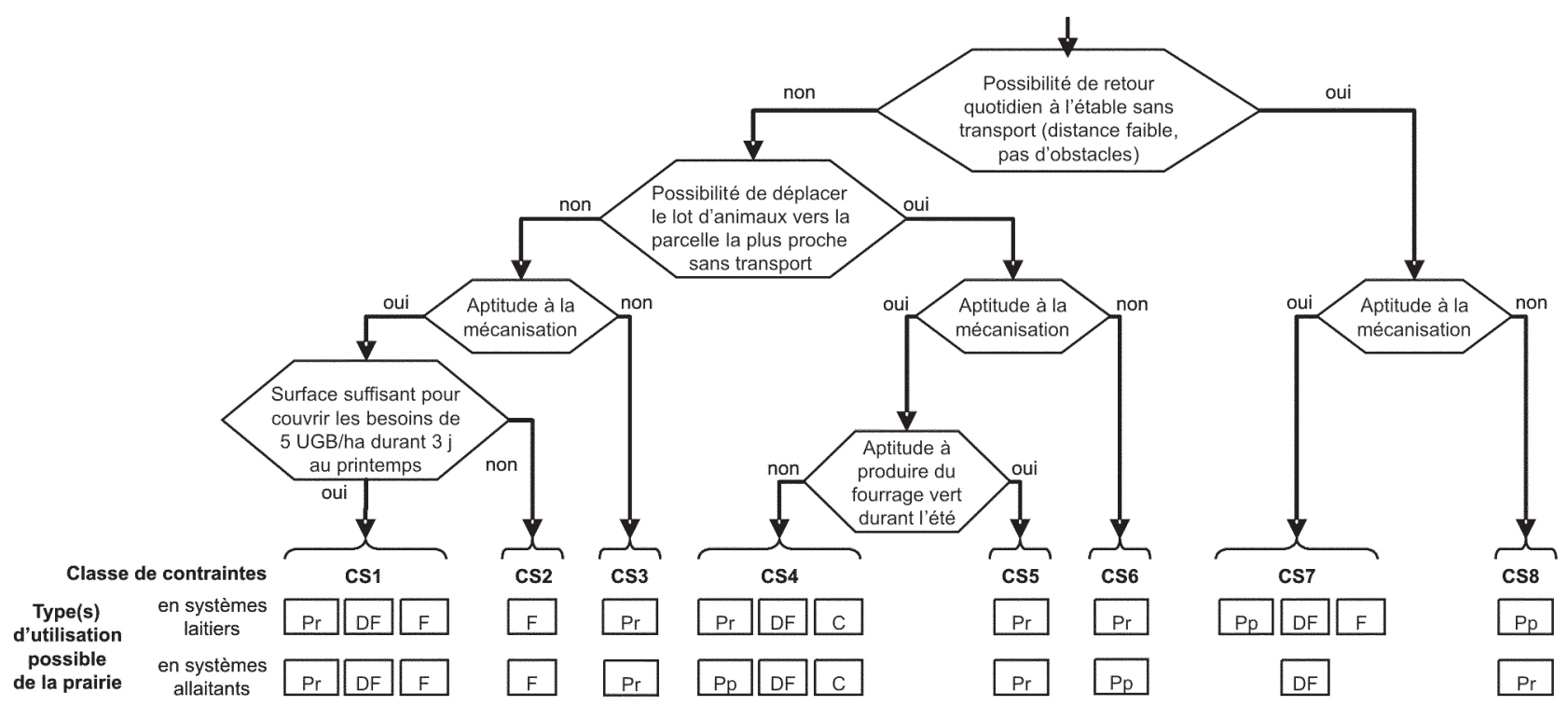

Cinq contraintes topographiques et d'exploitation sont listées dans un ordre hiérarchique (de haut en bas) et reliées pour former des ensembles de contraintes nommées "Classes de contraintes». Les combinaisons de contraintes qui n'avaient pas de sens pratiques ont été supprimées de l'arbre. Pour chaque classe de contraintes, un ou plusieurs types d'utilisation possible de la prairie au printemps sont spécifiés pour les systèmes bovins laitiers et allaitants. Les types d'utilisation sont : $\mathrm{Pp}$ : pâturage d'animaux en production ; $\mathrm{Pr}$ : pâturage d'animaux pour le renouvellement ; DP : pâturage (déprimage ou étêtage) suivi d'une fauche ; F : Fauche. Lorsque confronté au terrain, le type d'utilisation de 165 prairies correspond dans 139 cas au(x) type(s) d'utilisation possible fourni par l'arbre de discrimination. Cet arbre de discrimination permet de distinguer les parcelles à un type d'utilisation possible de celles qui offrent plusieurs possibilités, ces dernières constituant des sources de flexibilité organisationnelle.

seuils entraînant la sortie du système selon les conduites. Les modèles d'Andrieu et al (2008) et plus récemment de Martin (2009) sont centrés sur la question de la flexibilité des systèmes fourragers. Andrieu et al (2008) illustrent l'intérêt de la diversité des surfaces fourragères, liée à l'hétérogénéité du territoire de l'exploitation, pour limiter l'impact des aléas climatiques. Martin et al (2009) proposent de raisonner une «flexibilité potentielle» d'utilisation d'un couvert végétal en considérant par exemple le nombre de fonctions alimentaires que peut remplir une parcelle d'herbe selon un arbre de détermination (figure 3 ) et la plage d'utilisation optimale du couvert prairial selon ses traits fonctionnels. Des bases théoriques de raisonnements équivalents existent dans le domaine du troupeau, avec la compréhension de la plasticité du comportement animal à court et long terme (Blanc et al 2010) et de l'intérêt d'avoir une diversité de trajectoires productives animales. Force est de constater que les modèles de fonctionnement de systèmes d'élevage n'ont guère exploré les bases de la flexibilité potentielle et de la gestion adaptative des itinéraires techniques du troupeau.

\section{2 / Intérêts et limites pour l'analyse de la dynamique des systèmes d'élevage}

Les modèles de systèmes d'élevage évoqués dans cette partie explorent dans leur très grande majorité le comportement du fonctionnement de systèmes au sein de phases de "cohérence» ou de «maitrise croissante» du cycle adaptatif, et s'intéressent peu aux phases de reconfiguration de systèmes associés à des chocs. Seule l'approche de Doyen et Tichit (1999) explore les seuils d'irréversibilité en situation d'aléas et de chocs. D'une façon plus générale, les modèles référencés ici s'intéressent, dans leur majorité à la flexibilité opérationnelle, c'est-à-dire aux leviers de la résistance aux aléas dans le cadre d'un projet et d'une stratégie au contenu considéré comme constant. Autrement dit, la co-évolution du système et de son environnement demeure largement hors de portée des démarches actuelles (Coquil et al 2010). Notamment, l'apprentissage, l'acquisition de l'expérience et l'ajustement du pilotage au fur et à mesure du temps qui en découle est actuellement largement ignoré, tout comme les modalités d'une gestion adaptative qui remettrait en cause, selon les conditions de l'année, le projet de production animale. Or ce type de gestion adaptative stratégique se rencontre de façon non négligeable en fermes ainsi que nous l'avons montré précédemment.

Le contexte sous-jacent de développement des modèles est très souvent celui de l'exploitation spécialisée, à effectif animal stable, avec un projet de produc- tion raisonné autour de l'expression du potentiel. Nous avons vu dans la partie 2.1 que ces conditions ne reflètent que très partiellement les logiques d'action des éleveurs, lesquels intègrent souvent l'agrandissement, la combinaison d'activités comme principes pour durer, et parfois le maintien de sources de souplesses techniques. Les deux premiers cas de figure ne sont pas envisagés, ils nécessiteraient notamment d'y inclure un regard sur le travail et son organisation (Madelrieux et Dedieu 2008) et la façon dont la conduite peut être ajustée pour passer en travail. Le troisième cas relève de l'exploration des propriétés régulatrices de systèmes d'élevage que nous avons évoquée plus haut.

La modélisation est une simplification de la réalité. De fait, les modèles de systèmes d'élevage traitent rarement avec le même degré d'approfondissement de la gestion dynamique du troupeau (production, reproduction et renouvellement des capacités productives) et de celle des ressources, notamment fourragères. Du coup, il est assez difficile de rendre compte des implications sur le long terme d'ajustements conjoints de la conduite du troupeau et de celle des surfaces que l'on peut observer en ferme ou lors d'expérimentations système pour faire face à des aléas (Dedieu et al 2008c, Coquil et al 2009).

Si la flexibilité se décline relativement à des aléas spécifiés, force est 
alors de constater que les aléas climatiques et leurs conséquences sur l'alimentation des troupeaux monopolisent les recherches (comme aléas externes ; les aléas internes étant liés à la biologie de la reproduction). Or la volatilité des prix, qui ne cesse de croître, est pourtant aujourd'hui un facteur fort d'incertitude sur l'avenir, et d'interrogation sur les configurations même des systèmes. Mosnier et al (2009) ont développé des démarches d'optimisation permettant de raisonner, avec un point de vue économique, les choix d'activités en tenant compte de différents scénarios climatiques et de prix en élevage bovin viande. Cette approche, si elle ne rend pas compte du fonctionnement d'un système d'élevage soumis en continu à des aléas et des chocs, qui constitue la base de réflexion de cet article, mériterait d'être intégrée dans une réflexion plus large et pluridisciplinaire des conditions de la «flexi-sécurité» des systèmes d'élevage (Grasset 2008)

\section{Conclusion}

L'incertitude croissante sur les conditions de l'avenir et la recherche de systèmes qui «font avec» les aléas plutôt que de chercher à les gommer, implique d'accorder une grande attention aux capacités d'adaptation des systèmes d'élevage, à des échelles de temps (moyen et long terme) qui permettent de prendre en compte en continu les perturbations auxquelles ils sont soumis.

Les travaux présentées ici ne prétendent pas couvrir le vaste champ des recherches sur l'adaptation des systèmes d'élevage, ni proposer des résultats clés en main utilisables en l'état dans le cadre du conseil. Cependant, ce texte veut témoigner d'un axe de travail qui tend à se développer en France comme en Europe, alors que les recherches sur les systèmes en situation d'aléas étaient auparavant surtout nourries d'études de cas dans les pays du Sud ne disposant ni d'un climat tempéré, ni d'une politique agricole protectrice. Tant dans le domaine des études en fermes que dans celui de la modélisation et des expérimentations systèmes, les pistes d'amélioration des cadres conceptuels sont nombreuses. Nous n'avons qu'une vision très imparfaite, souvent limitée par les regards disciplinaires, des combinaisons de leviers mobilisés par les éleveurs pour résister aux aléas, et des conditions de réussite des transitions visant la reconfiguration de systèmes (Lamine et
Bellon 2009). Nos modélisations demeurent encore très statiques, ou fortement déséquilibrées entre d'une part des cadres décisionnels figés, sans apprentissage ni évolution d'une année sur l'autre, et d'autre part des fonctionnements biotechniques qui tendent à formaliser de mieux en mieux les interactions dynamiques, les arrières effets pour rendre compte des trajectoires productives animales, des évolutions des couverts végétaux et des ajustements des itinéraires techniques (Coquil et al 2010).

$\mathrm{Au}$ final, et pour synthétiser en quelques mots, la résistance aux aléas, aux chocs, l'aptitude à saisir de nouvelles opportunités pour reconfigurer un système s'appuie sur des énoncés simples (Darnhofer et al 2010) :

- préserver et renouveler la diversité des activités, des ressources, des trajectoires productives animales et même des normes sociales concernant l'élevage ;

- rechercher une flexibilité multisources (interne et externe) ;

- développer une capacité d'apprentissage, permettant l'accumulation et la mobilisation des expériences passées au service des décisions pour le futur.

\section{Références}

Aaker D., Mascarenhas B., 1984. The need for stratégic flexibility. J. Business Strat., 5, 74-82.

Andrieu N., Coleno F.C., Duru M., 2008. L'organisation du système fourrager source de flexibilité face aux variations climatiques. In : L'élevage en mouvement : flexibilité et adaptation des exploitations d'herbivores. Dedieu B., Chia E., Leclerc B., Moulin C.H., Tichit M. (Eds). Editions Quae, Paris, France, 95-110.

Astigarraga L., Chia E., Ingrand S., 2008. Production flexibility in extensive beef farming systems in the Limousin region. In: Enpowerment of the rural actors: a renewal of farming systems perspectives. Dedieu B., Zasser S., Bedoya S. (Eds). Proc. $8^{\text {th }}$ Eur. IFSA Symp., 6-10 July, Clermont Ferrand, France, CD Rom, 385-402.

Begon M., Pailleux J.Y., Joly N., Lemery B., Dedieu B., 2009. Les chemins pour durer en élevage bovin laitier : diversité des logiques d'action sur le long terme en Ségala (Massif Central). Renc. Rech. Rum., 16, 105-108.

Benoit M., 1998 Un outil de simulation du fonctionnement du troupeau ovin allaitant et de ses résultats économiques : une aide pour l'adaptation à des contextes nouveaux. INRA Prod. Anim., 11, 199-209.

Bernoux P., 1996. Les nouvelles approches sociologiques des organisations. Seuil, Paris, France, 244p.

Blanc F., Dumont D., Brunschwig G. Bocquier F., Agabriel J., 2010. Robustesse, flexibilité, plasticité : des processus adaptatifs révélés dans les systèmes d'élevage extensifs de ruminants. In : Robustesse, rusticité, flexibi- lité, plasticité, résilience... les nouveaux critères de qualité des animaux et des systèmes d'élevage. Sauvant D., Perez J.M. (Eds). Dossier Inra Prod. Anim., 23, 65-80.

Bertin J., 1977. La graphique et le traitement graphique de l'information. Flammarion. Paris, France, 273 p

Chardon X., Raison C., Le Gall A., Morvan T., Faverdin P., 2008. Fumigene: a model to study the impact of management rules and constraints on agricultural waste allocation at the farm level. J. Agric. Sci., 146, 521539 .

Chia E., 2008. La flexibilité relationnelle. Rôle des réseaux, groupements et associations d'éleveurs. In : L'élevage en mouvement : flexibilité et adaptation des exploitations d'herbivores. Dedieu B., Chia E., Leclerc B. Moulin C.H., Tichit M. (Eds). Editions Quae, Paris, France, 135-142.

Chia E., Marchenay M., 2008. Un regard des sciences de gestions sur la flexibilité : enjeux et perspectives. In : L'élevage en mouvement flexibilité et adaptation des exploitations d'herbivores. Dedieu B., Chia E., Leclerc B. Moulin C.H., Tichit M. (Eds). Editions Quae, Paris, France, 23-36.

Cialdella N., Dedieu B., 2010. What action logics do family livestock farmers have to maintain their activity over the long term? In Building sustainable rural future: The added value of systems approaches in times of change and uncertainty. 9eme IFSA Symp., Vienna, Autriche, 4-6 Juillet 2010. Accepté.
Coleno F.C., Duru M., Soler L.G. 2002. A simuation model of a dairy forage system to evaluate feeding strategies with spring rotational grazing. Grass Forage Sci., 57, 312-312.

Coquil X., Blouet A., Fiorelli J.L., Bazard C., Trommenschlager J.M., 2009. Conception de systèmes laitiers en agriculture biologique : une entrée agronomique. In : Numéro spécial, Elevage Bio. J.M. Perez (Ed). INRA Prod. Anim., 22, 221-234.

Coquil X., Dedieu B., Beguin P., 2010. How do livestock and crop sciences represent the evolution of farming systems. In: Building sustainable rural future: The added value of systems approaches in times of change and uncertainty. 9ème IFSA Symp., Vienna, Autriche, 4-6 Juillet 2010. Accepté.

Cournut S., Dedieu B., 2004. A discrete event simulation of flock dynamics: a management application to three lambings in two years. Anim. Res., 53, 383-403.

Cyrulnick B., 2001. Les vilains petits canards. Odile Jacob (Ed). Paris, France, 288p.

Darnhofer I., Bellon S., Dedieu B., Milestad R., 2010. Adaptiveness to enhance the sustainability of farming systems. A review. Agriculture for a sustainable development. Online DOI 10.1051/agro/2009053.

Dedieu B., 2009. Qualification of the adaptive capacities of livestock farming systems. Rev. Brasil. Zootech., 38, 397-404.

Dedieu B., Faverdin P., Dourmad J.Y., Gibon A., 2008a. Système d'élevage, un 
concept pour raisonner les transformations de l'élevage. In : Numéro spécial Anniversaire, 20 ans de recherches en productions animales à l'INRA. B. Charley, P. Herpin, J.M. Perez (Eds). INRA Prod. Anim., 21,45-58.

Dedieu B., Chia E., Leclerc B., Moulin C.H., Tichit M., 2008b. L'élevage en mouvement : flexibilité et adaptation des exploitations d'herbivores. Dedieu B., Chia E., Leclerc B., Moulin C.H., Tichit M. (Eds). Editions Quae, Paris, France, 294p.

Dedieu B., Louault F., Tournadre H., Benoit M., 2008c. Réponse des systèmes d'élevage innovants à la variabilité climatique : une expérimentation en production extensive ovin viande intégrant des préoccupations environnementales. In : L'élevage en mouvement : flexibilité et adaptation des exploitations d'herbivores. Dedieu B., Chia E., Leclerc B., Moulin C.H., Tichit M. (Eds). Editions Quae, Paris, France, 161-178.

Doyen L., Tichit M., 1999. A viability analysis of mixed herd management in uncertain climatic environments. Cahiers du centre de Recherche, Viabilité, Jeux Contrôle, 9906, 125.

Girard N., Lasseur J., 1997. Stratégies d'élevage et maîtrise de la répartition temporelle de la reproduction. Exemples en élevage ovin en montagne méditerranéenne. Cah. Agric., 6, 115-124

Grasset M., 2008. Adopter la flex-sécurité. Terra, 125.

Grossetti M., 2006. L'imprévisibilité dans les parcours sociaux. Cah. Int. Sociol., 120, 528.

Gunderson L.H., 2000. Ecological resilience in theory and practice. Ann. Rev. Ecol. Syst., 4, $1-23$.

Holling C.S., 1973. Resilience and stability of ecological systems. Ann. Rev. Ecol. Syst., 41, 1-23.

Holling C. S. 2001. Understanding the complexity of economic, ecological, and social systems. Ecosystems, 4, 390-405.

Ingrand S., Astigarraga L., Chia E., David C., Coquil X, Fiorelli J.L., 2009. Développer les propriétés de flexibilité des systèmes de production en situation d'incertitude ; pour une durabilité qui dure. Journ. Rech. Cunicole, $17-$ 18 novembre, Le Mans, France, Actes + CDRom 80-88.

Jouven M., Baumont R., 2008. Simulating grassland utilization in beef suckler systems to investigate the trade-offs between production and floristic diversity. Agric. Syst., 96, 260272.

Lamine C., Bellon S., 2008. Conversion to organic farming: a multidimensional research object at the crossroads of agricultural and social sciences. A review. Agron. Sustainable Dev., 29, 97-112.

Landais E., Bonnemaire J., 1996 La zootechnie art ou science ? Entre nature et socié- té, l'histoire exemplaire d'une discipline finalisée. Courr. Env., 27.

Lasseur J., Landais E., 1992. Mieux valoriser l'information contenue dans les carnets d'agnelage pour évaluer des performances et des carrières de reproduction en élevage ovinviande. INRA Prod. Amin., 5, 43-58.

Lev L., Campbell D., 1987. The temporal dimension in Farming sytems research: the importance of maintaining flexibility under conditions of incertainties. J. Rur. Stud., 3, 123-132.

Lemery B., Ingrand S., Dedieu B., Degrange B., 2005. Agir en situation d'incertitude : le cas des éleveurs bovins allaitants. Econ. Rur., 288, 57-69.

Levrouw F., Morales H., Arbeletche P., Malaquin I., Tourrand J.F., Dedieu B., 2007 Estrategias de largo plazo de los ganaderos uruguayos en situationces de incertitumbre. Agrociencia, 11, 87-93.

Madelrieux S., Dedieu, B., 2008 Qualification and assessment of work organisation in livestock farms. Animal, 2, 435-447.

Martel G., Dedieu B., Dourmad J.Y., 2008. Simulation of sow herd dynamics with emphasis on performance and distribution of periodic tasks events. J. Agric. Sci., 146, 365-380.

Martin G., 2009. Analyse et conception de systèmes fourragers flexibles par modélisation systémique et simulation dynamique. Thèse Université Toulouse, 173p.

Martin G., Hossard L., Theau J.P., Therond O., Josien E., Cruz P., Rellier J.P., MartinClouaire R., Duru M., 2009. Characterizing potential flexibility in grassland use. Application to the French Aubrac area. Agron. Sustainable Dev., 29, 381-389.

Mignon S., 2001. Stratégie de pérennité d'entreprise. Vuilbert, Paris, France, 232p.

Milestad R., Darnhofer I., 2003. Building farm resilience: the prospects and challenges of organic farming. J. Sustainable Agric., 22, 8197.

Mosnier C., Agabriel J., Lherm M., 2009. A dynamic bio-economic model to simulate optimal adjustments of suckler cow farm management to production and market shocks in France. Agric. Syst., 102, 77-88.

Moulin C.H., 1993. Le concept de fonctionnement de troupeau. Diversité des pratiques et variabilité des performances animales dans un système agropastoral sahélien. INRA Et. Rech. Sys. Agr. Dév., 27, 73-94.

Moulin C.H., Ingrand S., Lasseur J., Madelrieux S., Napoléone M., Pluvinage J., Thénard V., 2008. Comprendre et analyser les changements d'organisation et de conduite de l'élevage dans un ensemble d'exploitations : propositions méthodologiques. In : L'élevage en mouvement : flexibilité et adaptation des exploitations d'herbivores. Dedieu B., Chia E., Leclerc B., Moulin C.H., Tichit M. (Eds). Editions Quae, Paris, France, 181-196.
Osty P.L., 1978. L'exploitation agricole vue comme un système. Diffusion de l'innovation et contribution au développement, BTI, 326, 43-49.

Pasin F., Tchokogué A., 2001. La flexibilité multiforme des entreprises de transport. Rev. Fr. Gestion, Janvier-Février, 23-31.

Reix R., 1979. La flexibilité de l'entreprise. Editions Cujas. Paris, France, 180p.

Puillet L., 2010. Modéliser la variabilité biologique en réponse aux pratiques de conduite. Application au troupeau caprin laitier. Thèse, ABIES, 168p. + annexes.

Santucci P.M., 1991. Le troupeau et ses propriétés régulatrices, bases de l'élevage extensif. Thèse, Université Montpellier II, 85p.

Sauvant D., Martin O., 2010. Robustesse, rusticité, flexibilité, plasticité... les nouveaux critères de qualité des animaux et des systèmes d'élevage : définitions systémique et biologique des différents concepts. In : Robustesse, rusticité, flexibilité, plasticité, résilience... les nouveaux critères de qualité des animaux et des systèmes d'élevage. Sauvant D., Perez J.M. (Eds). Dossier Inra Prod. Anim., 23, 5-10.

Sebillotte M., Soler L.G., 1990. Les processus de décision des agriculteurs. . Modélisation systémique et système agraire : Décision et organisation. Actes du séminaire du département INRA de recherches sur les systèmes agraires et le développement. Brossier J., Vissac B., Le Moigne J.L. (Eds). St-Maximin, France, 93-117.

Tarondeau J.C., 1999. La flexibilité dans les entreprises. Collection Que sais-je ? PUF, $126 \mathrm{p}$.

Tichit M., Ingrand S., Moulin C.H., Cournut S., Lasseur J., Dedieu B., 2004. Analyser la diversité des trajectoires productives des femelles reproductrices : intérêts pour modéliser le fonctionnement du troupeau en élevage allaitant. Inra Prod. Anim., 17, 123-132.

Vayssieres J., Guerrin F., Paillat J.M., Lecomte P., 2009. Gamede: a global activity model for evaluating the sustainability of dairy entreprises. Part 1: whole farm dynamic model. Agric. Syst., 101, 128-138.

Veysset P., Bebin D., Lherm M., 2005. Adaptation to Agenda 2000 (CAP reform) and optimisation of the farming system of French suckler cattle farms in the Charolais area: a model-based study. Agric. Syst., 83, 179-202.

Walker B., Holling C.S., Carpenter S.R., Kinzig A., 2004. Resilience, adaptability and transformability in socio-ecological systems. Ecol. Soc., 9, 5 . 


\title{
Résumé
}

Comment rendre compte des capacités d'adaptation des systèmes d'élevage dans un environnement soumis en permanence à des perturbations dont certaines sont des chocs sévères? Nous proposons d'explorer cette question en mobilisant les concepts de résilience et de flexibilité et des travaux récents réalisés en ferme et en modélisation. Les enquêtes réalisées en France et en Uruguay (bovin lait et viande) suggèrent que le rapport à l'agrandissement, à la spécialisation et au fonctionnement (ambition technique, gestionnaire ou recherche de souplesse du système) différencient les logiques d'action sur le long terme de systèmes d'élevage résilients. Ces logiques d'action marquent également les leviers de la flexibilité opérationnelle c'est-à-dire de la résistance à plus court terme aux aléas climatiques et de prix. La flexibilité peut être externe dans les systèmes productifs mais techniquement tendus, interne dans des systèmes plus extensifs au profil de vente ajustable selon les conditions de l'année. La modélisation systémique s'est emparée des questions de capacités tampon, de gestion adaptative qui fondent les capacités d'adaptation et évolue vers l'exploration de systèmes conçus pour être flexibles. Elle demeure cependant cloisonnée d'un point de vue disciplinaire et est en difficulté pour aborder la co-évolution des systèmes et de l'environnement sur le long terme.

\begin{abstract}
Incertainties and adaptative capacities : theories and applications to the analysis of livestock farming systems dynamics

How can account be taken of the adaptive capacities of livestock systems in an environment that is permanently undergoing disruptions, some of which are shocks that bring the system itself into question? We propose to explore this question by mobilising the concepts of resilience and flexibility and consulting recent work carried out on farm and in systemic modelling. Surveys carried out in France and in Uruguay (dairy and beef cattle) suggest that the positions to enlargement, specialisation and functioning (technical or management ambition, or the search for system flexibility) differentiate the long term action logics of farmers whose livestock system shows resilience. These action logics also mark the levers of operational flexibility, i.e. resistance to short term hazards on climate and prices : flexibility is external in systems that are productive but technically strained ; internal in more extensive systems where the sales profile can be adjusted to conditions over the year. A review of recent French livestock farming system models show how systemic modelling deals with questions of buffer properties and adaptive management which establish the adaptive capacity, and explore flexible systems. But the models remain isolated from a disciplinary point of view and have difficulty in tackling the co-evolution of systems and the environment over the long-term.
\end{abstract}

DEDIEU B., INGRAND S., 2010. Incertitude et adaptation : cadres théoriques et application à l'analyse de la dynamique des systèmes d'élevage. In : Robustesse, rusticité, flexibilité, plasticité, résilience... les nouveaux critères de qualité des animaux et des systèmes d'élevage. Sauvant D., Perez J.M. (Eds). Dossier Inra Prod. Anim., 23, 81-90. 